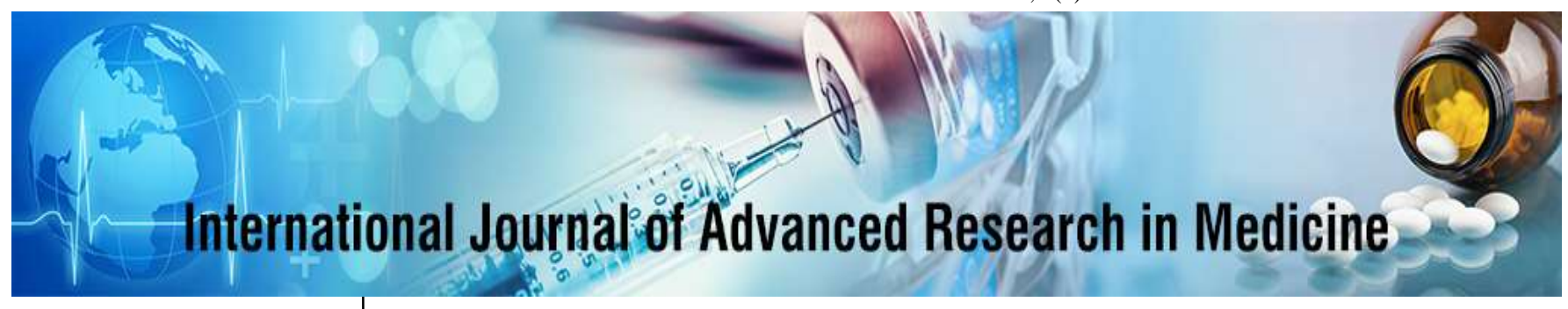

E-ISSN: 2706-9575

P-ISSN: 2706-9567

IJARM 2021; 3(1): 13-16

Received: 12-11-2020

Accepted: 16-12-2020

Dr. Pranay Kumar Patro Assistant Professor, Department of Medicine, S.C.B Medical College and Hospital, Cuttack, Odisha, India

\section{Dr. Ashok Kumar Behera} Assistant Professor, Department of Medicine, S.C.B Medical College and Hospital, Cuttack, Odisha, India

Dr. Aditya Narayan Sahu Assistant Professor,

Department of Medicine, S.C.B Medical College and Hospital, Cuttack, Odisha, India

Dr. Akash Rai Murmu Postgraduate Student, Department of Medicine, S.C.B Medical College and Hospital, Cuttack, Odisha, India
Corresponding Author: Dr. Pranay Kumar Patro Assistant Professor, Department of Medicine, S.C.B Medical College and Hospital Cuttack, Odisha, India

\section{Prognostic significance of hyperferritinemia in dengue: A tertiary health care centre study}

\author{
Dr. Pranay Kumar Patro, Dr. Ashok Kumar Behera, Dr. Aditya \\ Narayan Sahu and Dr. Akash Rai Murmu
}

DOI: https://doi.org/10.22271/27069567.2021.v3.i1a.90

\begin{abstract}
Background: Dengue infection is a major public health threat; early recognition is crucial to improve the survival in severe dengue. For prediction of dengue, various biomarkers are present but they are rarely used. We used serum ferritin to predict the severity at an earlier stage.

Methods: This is a prospective observational study conducted in a tertiary health care centre involving 200 dengue cases diagnosed by positive NS1 antigen by ELISA. Serum ferritin was measured in all at the time of diagnosis, day 3 and day 7 . We monitored the patients both clinically and biochemically daily and classified them as severe and non-severe according to $2009 \mathrm{WHO}$ criteria.

Results: The mean age of the patients was $34.14 \pm 12.4$ years. Out of 200 patients, $136(68.0 \%)$ patients were male and rest $64(32.0 \%)$ were females, 56 developed severe dengue. All the 200 patients had fever $(100 \%)$ and arthralgia $(100 \%)$. Other clinical symptoms like rash, nausea, abdominal pain, persistent vomiting, mucosal bleeding, liver enlargement, clinical fluid accumulation, dengue haemorrhagic fever, dengue shock syndrome, altered sensorium, severe plasma leakage, and patients with ALT more than $1000 \mathrm{U} / \mathrm{L}$ was reported in $108(54.0 \%), 58(29.0 \%), 46(23.0 \%), 59(29.5 \%), 32$ $(16.0 \%), 19(9.5 \%), 13(6.5 \%), 9(4.5 \%), 35(17.5 \%), 2(1 \%), 12(6.0 \%)$ and $3(1.5 \%)$ patients respectively. The serum ferritin level was correlated with patients having rash, abdominal pain and persisting vomiting both on day 3 and day 7. A negative correlation was observed between serum ferritin level and total platelets count both on day $3(\mathrm{r}=-0.2226 ; \mathrm{p}=0.0016)$ and day $7(\mathrm{r}=-0.3562$; $\mathrm{p}=0.0001$ ). Patients with severe dengue had significantly low platelet count and higher ferritin levels. Conclusions: Elevated serum ferritin levels can be used as a potential early prognostic marker to predict the severity of dengue infection in clinical practice.
\end{abstract}

Keywords: Predictor of severity, serum ferritin, early, severe dengue

\section{Introduction}

Dengue is a systemic and dynamic disease. It is a mosquito borne tropical infection that is caused by dengue virus ${ }^{[1]}$. Primarily Dengue virus has four serotypes DENV1, DENV2, DENV3, DENV4. It is a RNA virus belonging to Flaviviridae. The global burden of dengue has increased at least fourfold over last three decades and there are now 1.5 Billion people at risk of the disease but the case fatality rate in dengue is only $2.5 \%{ }^{[2]}$. Before 1970 only nine countries has experienced some dengue epidemics. But at present Dengue has affected more than 100 countries in tropical and subtropical countries. Today Dengue virus possess a major threat to global public health. The number of dengue outbreaks caused by four serotypes of dengue has increased since 1980, mainly affecting South America, Asia and the Carribean. It is transmitted primarily by the vector female Aedes ${ }^{[3]}$ aegypti through bite. The female mosquito bites human during day time. Once infected, the mosquito remains infective for life time. Clinically severe dengue results from interplay between virus related virulence factors and host factors which include inflammatory response of the host to infection with exuberant $\mathrm{T}$ and $\mathrm{B}$ cell activation, release of cytokines (cytokine storm), altered endothelial function with increased vascular permeability and nutritional status of the host ${ }^{[4]}$.

Dengue infections result in dramatic alterations of host physiological parameters and there could be many potential biochemical and immunological markers ${ }^{[5,6]}$ which might have diagnostic and prognostic value. Serum Ferritin is an acute phase protein and elevated levels of Ferritin have been associated with the pathogenesis of many inflammatory, infectious viral diseases like Hepatitis C, Dengue Fever, West Nile fever.

One study in India showed that $\alpha-1$ Antitrypsin, Ceruloplasmin and Ferritin was raised in dengue fever. Serum Ferritin have longer half-life unlike the cytokines, so that it is easy to measure. 
This present study was carried out with an aim to establish a relation between Serum Ferritin and the severity of Dengue fever in adult population.

\section{Materials and Method}

This is a hospital based prospective observational study conducted from January 2017 to September 2018 in a tertiary health care center i.e S.C.B Medical College, Cuttack, Odisha. All probable cases above 15 year of age with fever, aches, rash, hemorrhagic rash admitted to the Hospital were taken in to consideration.

\section{Inclusion criteria}

- Adults population of $>15$ years of age with fever, aches, rash, abdominal pain, vomiting.

\section{Exclusion criteria}

\section{- Pediatric population}

- Patients with chronic diseases like, Malaria, Menningitis, enteric fever, Iron deficiency anaemia, collagen vascular diseases, immunocompromised state, Multiple Blood Transfusion, Liver diseases, Diabetes melitus and malignancy

- Patients did not give consent for the study.

After obtaining patients informed consent for participation in the study, $5 \mathrm{ml}$ of venous blood samples was collected. NS1Ag test was carried out by ELISA methods and serum ferritin level was analysed by using electrochemiluminescence immunoassay. Serum Ferritin level was measured twice i.e. on day $3^{\text {rd }}$ and on day $7^{\text {th }}$ for comparison and analysis.

Total 200 patients were taken for the study who fulfill the criteria for the purpose. The severity of dengue fever was classified by clinical features and blood parameters according to WHO classification 2009 for dengue fever.

\section{Statistical analysis}

The data was entered in a predesigned excel sheet for window. The data was presented by number followed by percentage and mean followed by standard deviation etc. The comparison of mean between two groups was carried out by Mann-Whitney test. The comparison of mean of serum Ferritin level at day 3 and day 7 was compared with paired $\mathrm{t}$ test. The correlation coefficient was calculated between serum Ferritin level and total platelets counts of the patients. A p value $<0.05$ was considered for statistical significant. All the data was analysed by using Graph-Pad Instat version 3 for window.

\section{Results}

Two hundred dengue positive confirmed patients were considered for final analysis. The mean age of the patients was $34.14 \pm 12.4$ years. The age of the patients was ranging from 15 years to 79 years. Out of 200 patients, $136(68.0 \%)$ patients were male and rest $64(32.0 \%)$ were females. There was no difference in the mean age of the male and females patients $(p>0.05)$.

The patients were presented with various clinical signs and symptoms. All the 200 patients had fever (100\%) and arthralgia (100\%). Other clinical symptoms like rash, nausea, abdominal pain, persistent vomiting, mucosal bleeding, liver enlargement, clinical fluid accumulation, dengue haemorrhagic fever, dengue shock syndrome, altered sensorium, severe plasma leakage, and patients with ALT more than $1000 \mathrm{U} / \mathrm{L}$ was reported in 108 (54.0\%), 58 (29.0\%), 46 (23.0\%), 59 (29.5\%), 32 (16.0\%), 19 (9.5\%), 13 $(6.5 \%), 9(4.5 \%), 35(17.5 \%), 2(1 \%), 12(6.0 \%)$ and 3 $(1.5 \%)$ patients respectively. The distribution of patients on the basis of their clinical signs and symptoms has been illustrated in Table-1

The serum ferritin level was measured at day 3 and day 7 of each patients. The median serum ferritin level at day 3 was found to be $909 \mathrm{ng} / \mathrm{mL}$ and at day 7 was $812 \mathrm{ng} / \mathrm{mL}$. There was no statistical significant difference in mean serum ferritin level measured at day 3 and day 7 (Paired t test, 0.7067; $\mathrm{p}=0.4846$ ). The serum Ferritin level was compared in patients with and without symptoms. There was a significantly high value of serum ferritin level in patients with rash, abdominal pain and persistent vomiting in comparision to patients without these symptoms both on day 3 and day 7. The detail comparison of serum ferritin level among the patients with and without symptoms measured at day 3 and day 7 has been shown in table- 2 .

The serum ferritin level was correlated with total platelets level both at day 3 and day 7. A negative correlation was observed between serum ferritin level and total platelets level both at day $3(\mathrm{r}=-0.2226 ; \mathrm{p}=0.0016)$ and day $7(\mathrm{p}=-$ $0.3562 ; \mathrm{p}=0.0001$ ). The correlation between serum ferritin level and total platelets level both at day 3 and day 7 was presented in Figure 1 and Figure 2 respectively.

Table 1: Distribution of patients on the basis of their clinical signs and symptoms $(n=200)$

\begin{tabular}{|c|c|}
\hline Presentation & No. of cases (\%) \\
\hline Fever & $200(100 \%)$ \\
\hline Arthralgia & $200(100 \%)$ \\
\hline Rash & $108(54 \%)$ \\
\hline Nausea & $58(29 \%)$ \\
\hline Abdominal Pain & $46(23 \%)$ \\
\hline Persistent Vomiting & $59(29.5 \%)$ \\
\hline Mucosal Bleeding & $32(16 \%)$ \\
\hline Liver Enlargement $>$ cm & $19(9.5 \%)$ \\
\hline Clinical Fluid Accumulation & $13(6.5 \%)$ \\
\hline Dengue Haemorrhagic Fever & $9(4.5 \%)$ \\
\hline Dengue Shock Syndrome & $35(17.5 \%)$ \\
\hline Altered Sensorium & $2(1 \%)$ \\
\hline ALT>1000 & $3(1.5 \%)$ \\
\hline Severe Plasma Leakage & $12(6 \%)$ \\
\hline
\end{tabular}

Table 2: Comparison of serum ferritin level among the patients with and without symptoms measured at day 3 and day 7 .

\begin{tabular}{|c|c|c|c|c|c|c|}
\hline \multirow{2}{*}{ Clinical symptoms } & \multicolumn{2}{|c|}{ Serum Ferritin (ng/mL) (Day 3) } & P value & \multicolumn{2}{|c|}{ Serum Ferritin (ng/mL) (Day 7) } & P value \\
\cline { 2 - 7 } & Present & Absent & & Present & Absent & \\
\hline Rash & $1778.4 \pm 1802.3$ & $988.5 \pm 756.3$ & 0.0001 & $1766.3 \pm 1917.8$ & $967.9 \pm 844.4$ & 0.0001 \\
\hline Abdominal pain & $2047.6 \pm 1804.8$ & $1217.8 \pm 1289.1$ & 0.0001 & $2020.8 \pm 2075.5$ & $1193.1 \pm 1323.3$ & 0.0001 \\
\hline Persistent vomiting & $2031.9 \pm 1785.2$ & $1172.8 \pm 1234.9$ & 0.0001 & $1973.1 \pm 1873.6$ & $1177.1 \pm 1379$. & 0.0001 \\
\hline
\end{tabular}




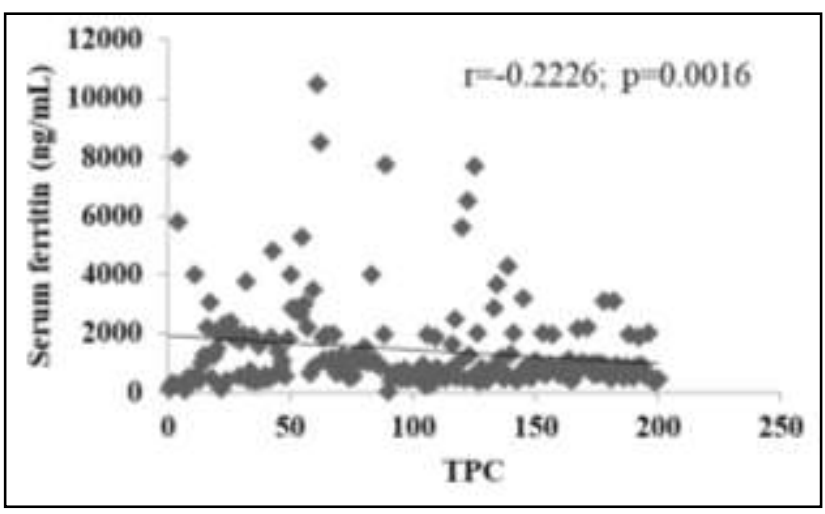

Fig 1: Correlation between serum ferritin level and total platelets level at day 3

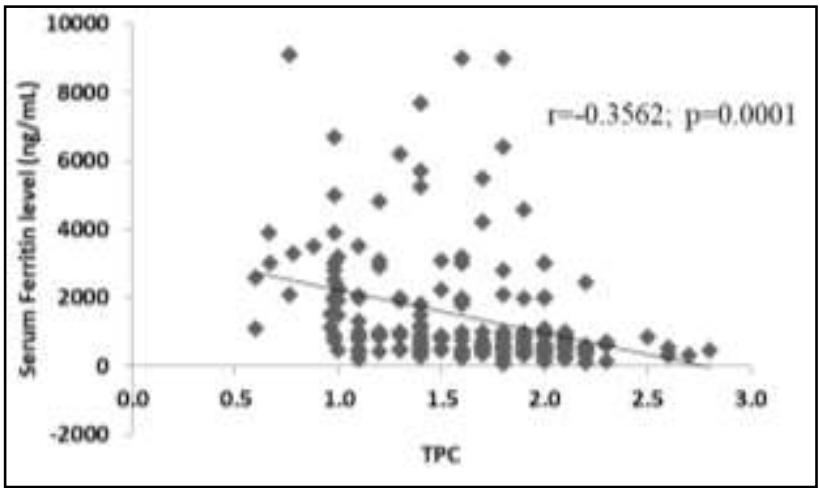

Fig 2: Correlation between serum ferritin level and total platelets level at day 7

\section{Discussion}

A total of 200 patients were Dengue positive were considered in this study. Out of 200 patients, majority were males (136 males and 64 females). In a study conducted in Singapore showed that among all PCR positive Dengue fever cases $71 \%$ were male ${ }^{[12]}$. Thai et al. ${ }^{[10]}$, in 2011 found that mostly young adults were affected with dengue fever. In male $51(37.5 \%)$ had dengue fever without warning signs, $44(32.4 \%)$ with warning signs and $41(30.1 \%)$ had severe dengue. In female cases $33(51.6 \%)$ had dengue fever without warning signs, $16(25 \%)$ with warning signs and 15 (23.4\%) had severe dengue. It was seen that maximum patients had dengue fever without warning signs. Hepatomegaly $19(9.5 \%)$, clinical fluid accumulation 13 $(6.5 \%)$, severe plasma leakage $12(6 \%)$ was seen. High ALT (>1000 IU/L) was present in $3(1.5 \%)$ cases. There were 9 $(4.5 \%)$ and $35(17.5 \%)$ cases of dengue haemorrhagic fever and dengue shock syndrome respectively. We observed 84 (42\%) patients were having dengue fever without warning signs, $60(30 \%)$ patients with warning signs and $56(28 \%)$ patients had severe dengue.

On day 3, the Mean serum Ferritin level in patients having rash, abdominal pain and persistent vomiting were $1778.4 \pm$ 1802.3 , $2047.6 \pm 1804.8,2031.9 \pm 1785.2$ respectively, which is significantly high compared to patients without these symptoms i.e. $988.5 \pm 756.3,1217.8 \pm 1289.1,1172.8$ \pm 1234.9 respectively. Similarly, on day 7 , serum Ferritin levels were very high in patients having warning symptoms in comparison to without symptoms. The serum ferritin level was significantly high in patients having warning signs both on day 3 and day 7 . These findings are in concordance with the study patients in Wathanee et al. (2008) in Bangkok ${ }^{[7]}$. Serum ferritin is an acute phase reactant and is frequently elevated during inflammatory or infectious conditions. The cytokines released in severe form causes raise in cellular ferritin by induction of the $\mathrm{H}$ chain of ferritin both transcriptionally and post-transcriptionally. The study conducted by Soundravally et al. concluded that hyperferritinemia could predict the Dengue severity with sensitivity of $76.9 \%$ [6]. A study conducted in Aruva in Brazil showed that increased levels of Ferritin are significantly associated with dengue viral disease severity [11].

There was significant thrombocytopinea in patients having high serum Ferritin level. These findings are in concordance with Jyothi S et al. $2018^{[13,14]}$.

Hyper-ferritinemia in dengue fever is associated with elevation of SGOT, SGPT and serum creatinine. A very strong correlation exist between $3^{\text {rd }}$ day serum ferritin and SGOT $(r=0.807, p<0.001)$, serum creatinine $(r=0.592$ and $p<0.001)$ and also between $3^{\text {rd }}$ day serum ferritin and SGPT $(\mathrm{r}=0.783, p<0.001)$. Similar result was also seen in the paper on dengue infection in the Aruba Islands by Brazilian and the Dutch medical researcher for rise in SGOT and SGPT ${ }^{[9]}$. Nadeem et al. $2016^{[8]}$, showed hyper ferritinaemia in dengue fever indicates highly active disease resulting in immune activation. There occur reactive hepatitis and ischemic insult to hepatic cell in dengue shock syndrome, which causes rise in liver enzymes. Hepatocytes are also activated by proinflammatory cytokines to produce high amount of ferritin, an acute phase protein leading to immune activation in dengue.

\section{Conclusion}

Hyperferritinemia can be used to predict the severity of Dengue and also the prognosis in Dengue patients. So patients having high levels of serum ferritin should be monitored very carefully from the very beginning.

Conflict of Interest: All the authors declared that there is no financial conflict of interest.

\section{References}

1. World Health Organization. Working to Overcome the Global Impact of Neglected Tropical Diseases. First WHO report on Neglected Tropical Diseases 2010. Available at: www.who.int/publications/2010/9789241564090_en g.pdf. Accessed on 29 November 2018.

2. World Health Organization. Regional Office of SouthEast Asia. Dengue fact sheet. Available at: http://www.searo.who.int/entity/vector_borne_tropi cal_diseases/data/data_factsheet/en/. Accessed on December 3, 2018

3. Back AT, Lundkvist A. Dengue viruses-an overview. Infect Ecol Epidemiol 2013;3:10-13.

4. Martina BEE, Koraka P, Osterhaus ADME. Dengue virus pathogenesis: an integrated view. ClinMicrobiol Rev 2009;22:564-81.

5. John DV, Lin YS, Perng GC. Biomarkers of severe dengue disease-a review. J Biomed Sci 2015;22:83.

6. Soundravally R, Agieshkumar B, Daisy M, Sherin J, Cleetus $\mathrm{C}$, et al. Ferritin levels predict severe dengue. Infection 2015;43(1):13-19.

7. Wathanee C, Ampaiwan Chuansumrit et al. Dengue nonstructural protein 1 antigen in the urine as a rapid and convenient diagnostic test during the febrile stage 
in patients with dengue infection. Diagnostic Microbiology and Infectious Disease, Elsevier 2011;71(4):467-469.

10.1016/j.diagmicrobio.2011.08.020. pasteur01741101

8. Nadeem M, Shafiq MM, Manzoor MS, Ahmed SI. Journal of Rawalpindi Medical college 2016;20(3):165167.

9. Soumyabrata Roy Chaudhuri, et al. Serum Ferritin a backstage weapon in diagnosis of dengue fever: interdisciplinary Perspectives on Infectious Diseases 2017. Article ID 7463489.

10. Thai KTD, Nishiura H, Hoang PL, Tran NTT, Phan GT, Le HQ, et al. Age-Specificity of Clinical Dengue during Primary and Secondary Infections. PLoS Negl Trop Dis 2011;5(6):e1180.

https://doi.org/10.1371/journal.pntd.0001180

11. van de Weg CA, Huits RM, Pannuti CS, Brouns RM, van den Berg RW. Hyperferritinaemia in dengue virus infected patients is associated with immune activation and coagulation disturbances. PLoS Negl Trop Dis 2014;9:e3214.

12. Lee LK, Gan VC Lee VJ, Tan AS, Leo YS, Lye DC, et al. Clinical relevance and discriminatory value of elevated liver aminotransferase levels for Dengue severity. PLoS Negl Trop Dis 2012;6:e1676

13. Petchiappan V, et al. Can serum ferritin level predict the severity of dengue early ? An observational study. Int J Res Med Sci 2019;7(3):876-881.

14. Gokul K Bharathi, et al. An Obseronal Studyseru Fertin level in dengue fever and it's correlation with severity of dengue fever. sJr Med Sci 2019;10:630-634. 